\title{
The Trouble with Cognitive Subtraction
}

\author{
K. J. Friston, C. J. Price, P. Fletcher, C. Moore, R. S. J . Frackowiak, and R. J . Dolan \\ TheWel Icome Department of Cognitive Neurology, Institute of Neurology, Queen Square, London WC1N 3BG, United Kingdom
}

Received J anuary 18, 1996

In this paper we present a critique of pure insertion. Pure insertion represents an implicit assumption behind many (but not all) studies that employ cognitive subtraction. The main contention is that pure insertion is not valid in relation to the neuronal instantiation of cognitive processes. Pure insertion asserts that there are no interactions among the cognitive components of a task. It is possi ble to evaluate and refute this assumption by testing explicitly for interactions using factorial experimental designs. It is proposed that factorial designs are more powerful than subtraction designs in characterizing cognitive neuroanatomy, precisely because they allow for interactions and eschew notions like pure insertion. In particular we suggest that the effect of a cognitive component (i.e., an effect that is independent of other components) is best captured by the main (activation) effect of that component and that the integration among components (i.e., the expression of one cognitive process in the context of another) can be assessed with the interaction terms. In this framework a complete characterization of cognitive neuroanatomy includes both regionally specific activations and regionally specific interactions. To illustrate our point we have used a factorial experimental design to show that inferotemporal activations, due to object recognition, are profoundly modulated by phonological retrieval of the object's name. This interaction implicates the inferotemporal regions in phonological retrieval, during object naming, despite the fact that phonological retrieval does not, by itself, activate this region. 1996 Academic Press, Inc.

\section{INTRODUCTION}

This paper is a critique of cognitive subtraction as a conceptual framework used in the design of brain activation experiments. In particular we focus on serial subtraction designs that rely on the assumption of pure insertion. Serial subtraction designs are well established and powerful devices in mapping cognitive neuroanatomy (e.g., Petersen et al ., 1989) but are predicated on possibly untenable assumptions about the relationship between brain dynamics and the functional pro- cesses that ensue (and where these assumptions may be tenable they are seldom demonstrated to be so). Concerns with serial subtraction can be formulated in terms of the relationship between cognitive processes and their neuronal implementation. In this paper we suggest that nonlinear systems like the brain do not behave in a fashion this is consistent with cognitive subtraction. We illustrate our point with a simple example - the functional anatomy of phonological retrieval during object naming.

\section{Cognitive Subtraction, Pure Insertion, and Additive Factors}

Serial subtraction involves the successive elaboration of a task by adding separable cognitive components and measuring the resulting increases in neuronal activity elicited by these tasks (using for example regional cerebral blood flow-rCBF). The physiological activations, which obtain on serial subtraction of these measurements, are then identified with the added cognitive components. The approach, predicated on pure insertion, assumes that each cognitive component evokes an "extra" physiological activation that is the same irrespective of the cognitive or physiological context. Pure insertion is an idea that underlies the original Donders subtractive method and has proven itself in many situations; for example in the psychophysics of reaction time measurements during the detection of visual targets embedded in a background of distracters. The linear (additive) relationship between reaction time and the number of distracters has been used to infer a "serial search" of the visual field (e.g., Treisman et al., 1985). Compelling examples of pure insertion usually involve an empirical demonstration of this additive relationship between a perceptual or cognitive process and a phenomenological brain measure (e.g., reaction time). However pure insertion in the context of brain activation experiments is an a priori assumption that has not been validated in any physiological sense. We present here an evaluation, in physiological terms, of cognitive subtraction by focusing on pure insertion: This evaluation follows Sternberg's proposal (Sternberg, 1969) to use additivity and interaction within 
factorial designs (the additive factor method) to address this issue.

Pure insertion is implicit in cognitive subtraction. The idea is that as a new cognitive $(A)$ component is added to a task, the implementation of the preexisting components (e.g., B) remains unaffected. If this were not the case the difference between tasks that did, and did not, include component B would depend on the presence of component $A$. In other words pure insertion requires that one cognitive component does not affect the effect of another. In factorial designs pure insertion is another way of saying that the interaction terms are negligible. The fact that interactions can be measured, using functional imaging (Friston et al., 1992a), means that the validity of pure insertion can now be addressed empirically. In this paper we use a simple factorial design to demonstrate that the physiological brain does not conform to pure insertion.

\section{The Nonlinear Brain and Interactions}

Even if, from a functionalist perspective, a cognitive component can be added without interacting with preexisting components, the brain's implementation of these processes is almost certainly going to show profound interactions. This follows from the observation that neural dynamics are nonlinear (e.g., Aertsen and Preissl, 1991). Nearly all theoretical and computational neurobiology is based on this observation. The point being made here is that although a cognitive science model, describing the functions, may include serial and additive elements the implementation of those functions is not. Consequently the structure of the cognitive components (functional model) and the brain's physiological implementation are not isomorphic and the mapping of one onto the other is problematic. Put boldly, cognitive science may be an internally consistent discipline, but it has no necessary or defined relation to measurements of brain function. Cognitive subtraction makes some strong assumptions about this relationship which are difficult to reconcile with basic neurobiology.

One of the innumerable examples of nonlinear brain dynamics that confound cognitive subtraction is modulation, from classical neuromodulation to large-scale modulatory interactions between different cortical areas (e.g., Friston et al., 1995a). A particularly relevant example here is the modulatory role of attention on perceptual processing; for example the responsiveness of V5 to motion in the visual field (Zeki et al., 1991). It is likely that this responsiveness is enhanced by selectively attending to motion (see the compelling study of Corbetta et al., 1991). V5 activation therefore represents an interaction between visual motion and selective attention. Consider now an experiment in which visual motion is presented with and without selective attention for motion. The resulting difference in physiological activation of $\mathrm{V} 5$ would, in terms of cognitive subtraction, be attributed to selective attention for motion. This would be a fallacious conclusion because the differential responses of V5 represent an interaction between visual analysis of a particular attribute and selective attention for that attribute. In neuronal terms this might be described as a modulation of V5 responsiveness to motion in the visual field mediated by afferents from some higher order area. The fallacy would be revealed by repeating the experiment in the absence of visual motion. In this instance "selective attention for motion" should not activate V5 because there are no motion-dependent responses to modulate. This second experiment would demonstrate an interaction between selective attention for motion and "visual motion" using a factorial experimental design. The V5 examplehighlights the cl ose relationship between functional interactions (between different cognitive or sensorimotor components) and statistical interactions that can be inferred using factorial designs. It should besaid that we do not consider "attention" a cognitive component (although the "control" of attention can be) but our point is clearly illustrated by this example. Furthermore a motion stimulus may not be necessary to demonstrate this modulatory effect of attention; for example "imagined" motion could interact with selective attention in an analogous way. This sort of finding would speak to the intimate relationship between imagery and attention.

Similar conclusions have been reached in neuropsychology: A patient with acquired dyslexia without dysgraphia was found to have a deficit in accessing phonology from semantics and a mild deficit in attending selectively to components of compound visual stimuli. These deficits interacted to severely disrupt her ability to name the components of visual arrays, despite the fact she could name each component when presented in isolation (the symptom of attentional dyslexics). This example "highlights the importance of interactions between deficits as being a major contributory factor to some neuropsychological syndromes" (Price and Humphreys, 1993).

In summary, pure insertion discounts both functional and physiological interactions and ther efore represents a very restrictive precondition for cognitive subtraction.

\section{Cognitive Subtraction vs Factorial Designs}

In this paper we suggest that a more powerful approach to cognitive neuroanatomy is to consider interactions explicitly, both in terms of the cognitive model and in terms of the experimental design and analysis. This acknowledges that the conjunction or integration of two or more cognitive processes may require an active physical instantiation. For example naming an object involves object recognition, phonol ogical retrieval, and the integration of the two, where that integration calls upon separable brain processes. Put 
more simply, phonological retrieval influences object recognition, and vice versa, and these effects are physiologically measurable. This perspective requires a factorial design where the interaction term represents the nonadditive (i.e., nonlinear) physiological concomitants of naming a recognized object, which is independent of the activations produced by recognizing or naming alone. This is the example used below.

The paper is divided into two sections. Both sections use the same data to address the same question, "I s the inferotemporal region implicated in phonological retrieval during object naming?" Considerable evidence from neuroanatomy and unit-electrode recordings suggests that neurons, in the inferotemporal cortex of animals, respond selectively to specific objects in the visual field or have the appropriate responses to do so (e.g., Ungerleider and Mishkin, 1982; Desimone et al., 1984; Perret et al., 1988) with convergent evidence in man (e.g., Sergent et al., 1992). On the basis of this and other evidence it might be hypothesized that inferotemporal regions are functionally specialized for object recognition in man. Furthermore lesion studies in man have shown that the ability to name objects is impaired when the inferotemporal regions are damaged. For exampleDeRenzi et al. (1987) studied the neuropsychological correlates of inferior temporal ischemic damage. As well as being alexic, subjects were impaired on naming objects and photographs. This sort of evidence suggests that the integrity of the inferotemporal cortex may be necessary for phonological retrieval (among other things) in object naming.

The first section of this paper addresses the above question from the perspective of cognitive subtraction, the implications for experimental design, analysis, and the ensuing conclusion. The second section critically reevaluates this conclusion in the light of the foregoing reservations about pure insertion, and approaches the question using a factorial design. The conclusion reached in this section differed fundamentally with that based on cognitive subtraction. The data presented here are part of a larger experiment designed by one of us (C.J .P.). The specific comparisons and (re)formulation of the experimental design, described below, serve to illustrate the points being made in this paper. A full description of these experiments and the results will be presented separately.

\section{THE COGNITIVE SUBTRACTION APPROACH}

\section{Experimental Design}

Consider the problem of designing an experiment to identify brain areas selectively activated by phonological retrieval during object naming. The cognitive processes involved in this task include visual analysis, object recognition, phonological retrieval, and speech. Suppose that we are not concerned with the sensorimotor aspects of the task but wish to test the hypothesis that the inferotemporal regions are involved in both object recognition and phonological retrieval. In this case we want a series of tasks that, on successive subtraction, isolate these two cognitive components (i.e., three tasks). The tasks used were:

(A) saying "yes" when presented with a colored shape (visual analysis and speech),

(B) saying "yes" when presented with a colored object (visual analysis, speech, and object recognition), and

(C) naming a visually presented colored object (visual analysis, speech, object recognition, and phonological retrieval).

Subtraction of task A from task B should identify brain regions associated with object recognition, and subtraction of task B from task C should identify regions implicated in phonol ogical retrieval. The hypothesis here is that both subtractions should activate the left infer otemporal regions (among other regions).

\section{Data Acquisition and Analysis}

The data were obtained from six subjects scanned 12 times (every $8 \mathrm{~min}$ ) while performing one of four different tasks (the three tasks A, B, C, and a further task $D$ to be described below). Scans were obtained, in pseudorandom order, with a CTI PET camera (Model 953B; CTI , Knoxville, TN). ${ }^{15} \mathrm{O}$ was administered intravenously as radiolabeled water infused over $2 \mathrm{~min}$. Total counts per voxel during the buildup phase of radioactivity served as an estimate of rCBF. Full details of the tasks will be presented elsewhere but in brief subjects were shown colored pictures of nonregular shapes or common objects on a VDU every $2 \mathrm{~s}$. The subjects were instructed to respond with either "yes" (tasks A and B) or a name (tasks C and D). To facilitate intersubject pooling, the data were realigned, spatially normalized (Friston et al., 1996), and smoothed with an isotropic 16-mm Gaussian kernel.

The data were analyzed using statistical parametric mapping (Friston et al., 1995b). We used a blocked one-way ANCOVA with global activity as a (subjectspecific) confounding covariate. Regionally specific differences were assessed using linear compounds (or contrasts) of effects due to the three replications of each task. Each contrast produces a statistical parametric map (SPM) of the t statistic. The SPMs were thresholded at $\mathrm{P}=0.05$ (uncorrected). In this paper we are only concerned with differential responses in the left inferotemporal region. Therefore we made no correction for multiple nonindependent comparisons in other brain regions.

\section{Results}

Significant $(P<0.05$ uncorrected) voxels from the SPMs were rendered onto a drawing of the left cortical 

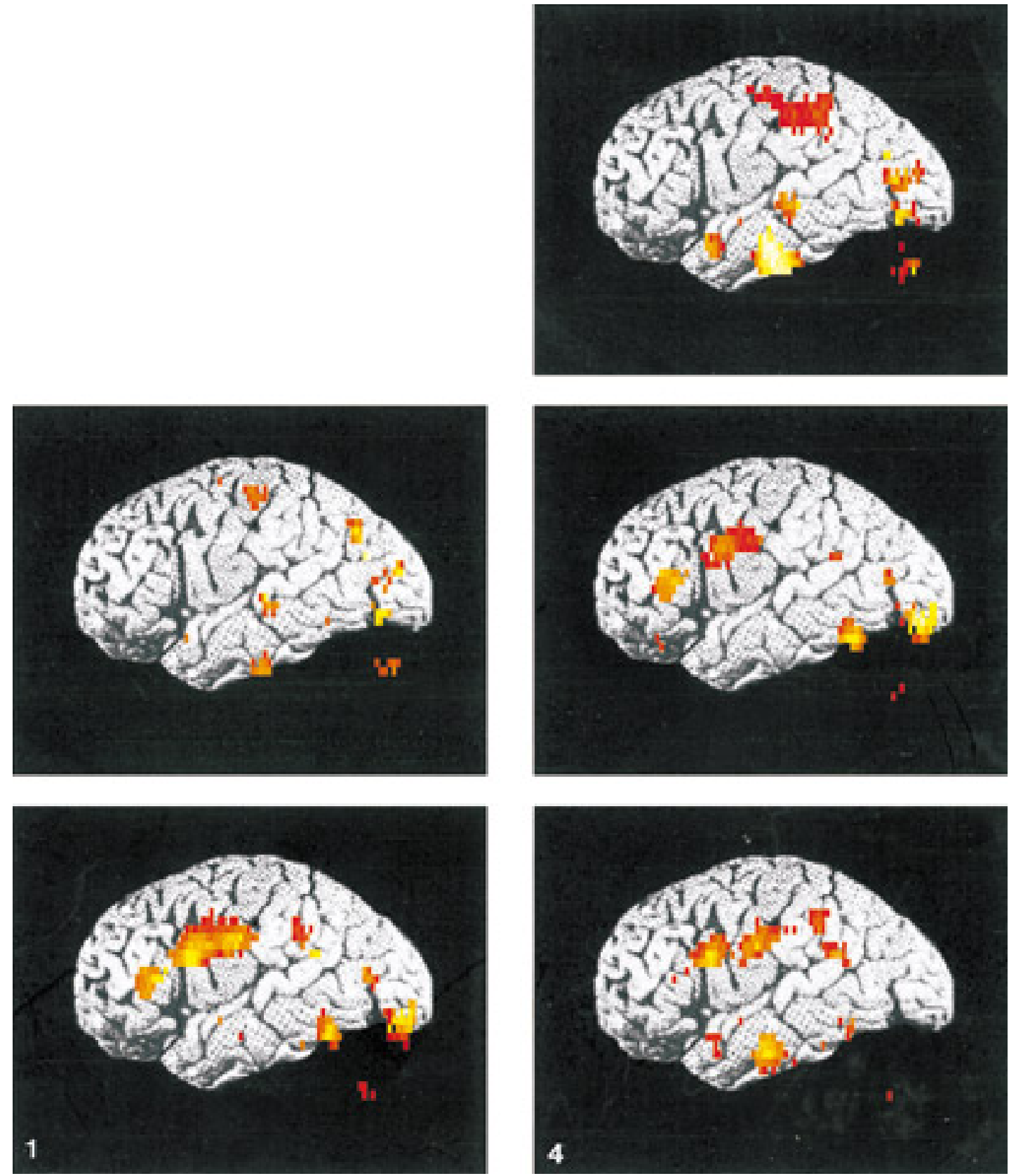

FIG. 1. Cognitive subtraction SPMs. These are a maximum-intensity projection of SPMs rendered onto a drawing of the left lateral cortical surface. Only voxels significant at $\mathrm{P}<0.05$ (uncorrected) are shown. The color scale is arbitrary and the space conforms to that described in the atlas of Talairach and Tournoux (1988). (Top) SPM representing the subtraction of task A from task B to reveal activations due to object recognition. (Bottom) Subtraction of task B from task C characterizing activations due to phonol ogical retrieval. These SPMs were predicated on a cognitive subtraction model.

FIG. 4. Factorial analysis SPMs. These SPMs use the same format as Fig. 1 and depict the main effects (activations) and interactions due to object recognition and phonological retrieval. (Top) Main effect of object recognition [i.e., $(B+C)-(A+D)$ ]. (Middle) Main effect of phonological retrieval [i.e., $(D+C)-(A+B)$ ]. (Bottom) Interaction [i.e., $(C-D)-(B-A)$ ].

surface. The SPM representing the subtraction of task A from task B is shown in Fig. 1, top, and includes, as predicted, a small focus in the left inferotemporal region. Other areas identified in this comparison include extrastriate regions, the superior temporal sulcus and angular gyrus, and the cerebellum (isolated focus on the lower right). However, we make no statistical inference about these effects because we did not use corrected $P$ values. Figure 1 (bottom) shows the second subtraction comparing tasks $B$ and $C$. This SPM shows 
activation foci in the extrastriate regions, ventrolateral occipitotemporal region, angular gyrus, prefrontal regions, and cerebellum. Again we make no statistical inferences about these activations in this paper. A separate communication will discuss these results in the light of the appropriate a priori hypotheses. In this paper we focus only on the inferotemporal region. From the current perspective the key thing to note is that the inferotemporal regions show no further activation due to phonological retrieval. On the basis of these results one might conclude that the inferotemporal region is specialized for (implicit) object recognition and that this cognitive component is sufficient to explain the activations even in the context of naming that object. In other words there is no evidence for differential responses in the inferotemporal regions due to phonological retrieval. Figure 2 shows the activity of a voxel in the inferotemporal region $[-48,-24,-32 \mathrm{~mm} \mathrm{BA}$ 20 -according to the atlas of Talairach and Tournoux (1988)] during the three tasks (A, B, and C). These results show that phonological retrieval does not, in itself, activate this region. The conclusion is that the inferotemporal regions cannot be implicated in phonological retrieval (as far as can be measured with PET).

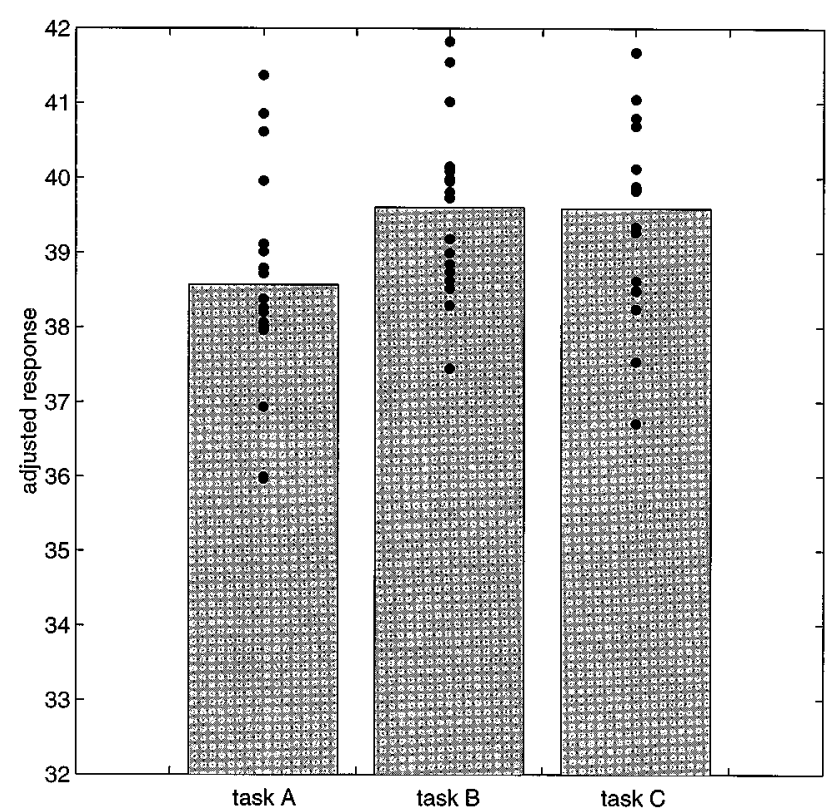

FIG. 2. Inferotemporal responses to task-dependent activities. Adjusted (for the confounding effects of global activity and the subject or block effect) rCBF equivalents for the three tasks ( $A, B$, and $\mathrm{C}$ ). The bars represent mean activity and the dots are the individual data points from each scan. Note that there is activation in passing from task $A$ to $B$ but not in going from task $B$ to $C$. These data were taken from a voxel in the left inferotemporal region $(-48,-18,-24 \mathrm{~mm}$ according to the atlas of Talairach and Tournoux).

\section{A CRITICAL REEVALUATION}

\section{Experimental Design}

The for egoing conclusion is wrong because it assumes pure insertion: Namely, that the activation due to object recognition is the same, irrespective of whether phonological retrieval is present or not. In order to say that phonological retrieval does not activate the inferotemporal region (according to the second subtraction) one has to assume that the activation due to object recognition is the same as in the first subtraction (i.e., object recognition in the absence of phonological retrieval). To validate this assumption we need to measureactivation due to object recognition in the presence of phonol ogical retrieval. This can be effected by comparing tasks that involve phonological retrieval with and without object recognition. This comparison required the fourth condition:

(D) Name the color of a presented shape (visual analysis, speech, and phonol ogical retrieval ).

Subtraction of task $A$ from task $B$ gives the activation due to object recognition in the absence of phonological retrieval, and subtraction of task $D$ from $C$ gives the recognition-dependent activation in the context of phonological retrieval. Pure insertion requires these activations to be the same and this is not the case. Figure 3 (top) shows that the activation in the context of phonological retrieval is far greater than under conditions without phonological retrieval. In other words phonological retrieval can be thought of as modulating the recognition-dependant responses of the inferotemporal region and in this sense the inferotemporal regions contribute to phonological retrieval.

There is an alternative and equival ent per spective on this interaction, which considers the inferotemporal activations induced by phonological retrieval with and without object recognition. Figure 3 (bottom) shows that, in the absence of object recognition, phonological retrieval deactivates the inferotemporal regions, whereas in the context of object recognition, this effect is nullified if not reversed. These data come from a voxel in BA $20(-48,-18,-24 \mathrm{~mm})$. In summary, inferotemporal responses do discriminate between situations where phonological retrieval is present or not and can be directly implicated in this cognitive process. These differential responses are expressed at the level of interactions and were revealed only with a factorial experimental design.

The final problem addressed in this paper is how to characterize these physiological effects in a way that meaningfully relates to the underlying cognitive components. The problem here is that if the activation due to cognitive component A (e.g., object recognition) depends on whether other cognitive components are present or not (e.g., phonological retrieval), what is the effect of $A$ 

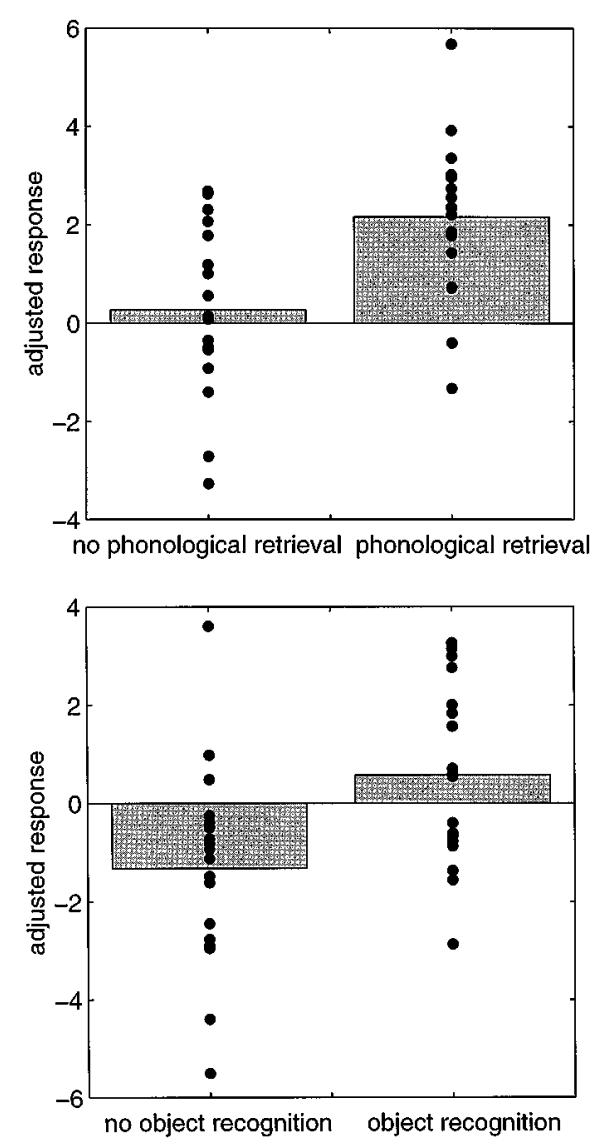

FIG. 3. Modulation of task-dependent activations. (Top) The activation due to object recognition with (right) and without (left) phonological retrieval. For this graph the difference was obtained by subtracting the adjusted activities in the first, second, and third occurrence of the two tasks in each subject. (Bottom) The same but comparing the activation due to phonological retrieval with (right) and without (left) object recognition. As in Fig. 2, the bars represent mean activity and the dots are the individual data points from each scan.

that is independent of these other components? One answer (in the context of a factorial design) is the main effect of $A$. This is simply the difference between all tasks that contain A and those that do not. In a two-by-two factorial design there are two main effects (due to the two components) and an interaction (due to the integration of these components). With this framework in mind we now present a factorial analysis of the data described in the previous section.

\section{A Factorial Analysis}

Using the same ANCOVA as in the previous section we tested for the main effect of object recognition, the main effect of phonological retrieval, and the interaction between them using the appropriate contrasts. The resulting SPMS are shown in Fig. 4. The top represents the main effect of object recognition and, as above, includes the inferotemporal regions but now the spatial extent is much greater. Comparison with Fig. 1 reveals an additional anterior temporal focus. Note that because we used the same statistical model, the statistical power (i.e., the degrees of freedom associated with the error term) is identical to the subtraction analysis. The middle shows the main effects of phonological retrieval. The final SPM (bottom) depicts the interaction (augmented activation) due to the conjunction of phonological retrieval and object recognition. This SPM clearly highlights the inferotemporal region and allows us to confirm that this region is implicated in phonological retrieval during object naming. It is interesting to note that some of the regions implicated in phonological retrieval (notably the angular gyrus) also show significant interactions. Because they are orthogonal (independent) the probability of main effects and interactions occurring in the same place, by chance, is exceedingly low. These findings will be discussed in a subsequent paper.

In this paper we have shown the difference between the two simple main effects (e.g., the main effect of object recognition without phonological retrieval and the main effect of object recognition with phonological retrieval) to illustrate the exact nature of the interaction (see Fig. 3). It is both usual and useful to report simple main effects when an interaction is significant, because the simple main effects characterize the exact nature of the interaction.

\section{DISCUSSION}

In this paper we have presented a deliberately emphatic critique of cognitive subtraction and in particular the notion of pureinsertion upon which it relies. The main contention is that pure insertion may, or may not, be a valid cognitive science level description, but it is almost certainly not valid in relation to the brain's implementation of cognitive functions. This follows from the fact that the brain is a highly nonlinear system and, phenomenologically, does not conform to additive or linear principles. We have used modulation, in the brain, to highlight the relationship between nonlinear neuronal interactions and statistical interactions in factorial experimental designs. Pure insertion disallows any interactions and yet these interactions are evident, even in the simplest experiments. To illustrate this point we have used a factorial experiment designed to elucidate the functional anatomy of object recognition and phonol ogical retrieval. We showed that pureinsertion can bean inappropriate and misleading assumption. In so doing we were able to demonstrate that inferotemporal activations, due to object recognition, were profoundly modulated by phonological retrieval of the object's name. This interaction clearly implicates the inferotemporal regions in phonological retrieval during object naming, despite the 
absence of a main effect of phonological retrieval in this region. By avoiding cognitive subtraction and using a factorial design of this sort we were ableto reconcile our functional imaging results with lesion deficit studies (e.g., DeRenzi et al., 1987).

This paper is not questioning the validity of cognitive subtraction. It questions the validity of pure insertion in relation to cognitive subtraction. The analysis of regionally specific effects, even in factorial designs, relies upon subtraction. For example, the interaction is equivalent to a difference between simple main effects and can be characterized as such. A simple main effect is, in most designs, a subtraction. Indeed if an interaction is demonstrated it is usual to characterize the nature of the interaction by reporting the simple main effects. It may be thought that the use of subtraction implies pure insertion, but it is not a necessary assumption. With careful experimental design and analysis one can eschew pure insertion. In many instances a factorial design facilitates this.

It is interesting to speculate that cognitive processes themselves may only express themselves at the level of interactions. For example the semantics (memory or knowledge of the meaning) of a word may only be realized in the interaction, or integration, between the word's phonology and the associated percept, intention, affect, or action (e.g., for nouns, the interaction between phonology and object recognition). If this is the case there would be no "semantic center" per se, but semantics would be subtended by interactions between a set of cortical regions subserving subordinate components (e.g., phonological retrieval and object recognition). In this regard it is noteworthy that in semantic dementia, which is associated with progressive loss of semantic knowledge, the atrophy seen on magnetic resonance images may be maximal in the inferolateral temporal cortex (Hodges et al., 1992). Although very conjectural this perspective is consistent with conclusions based on lesion data. For exampleAlexander et al. (1989) discuss the distribution of cortical lesions associated with transcortical sensory aphasia and consider the left inferior temporal lobe [see also Nielsen (1946)], the posterior inferior parietal lobe, and the white matter connections between these regions as part of a distributed system dealing with semantic representations.

We reiterate that the task analysis (decomposition of tasks into separable processing components) employed in this paper served to illustrate a point and would normally require a more comprehensive analysis (e.g., behaviorial or psychological justification for this particular decomposition and the equivalence of the different components over tasks). For example it may be said that the experimental design used here is not ideal, in that naming a color and naming an object do not represent the same cognitive component (or in statistical terms the level of a cognitive factor). However, this essentially is the point being made: Phonological retrieval only has real meaning in relation to the context (i.e., what is named). In the same sense object recognition is only completely specified when the context of this recognition is defined (e.g., recognition is implicit in task B and explicit in task C). This specification speaks directly to the interaction between the cognitive component and the context in which it is employed. In turn this interaction requires a factorial design for proper characterization. Clearly a comprehensiveanalysis of object recognition would require a universe of experiments over a wide range of "contexts." It is therefore important to acknowledge explicitly that the main effects and interactions pertain to the tasks employed in the experiment. The sort of generalization implicit in cognitive subtraction to all contexts is probably unwarranted. In general while task analysis should guide the selection of tasks, the formulation of factorial designs necessitates the careful manipulation of task and instruction sets themselves.

We have previously described a broad taxonomy of experimental designs in functional imaging (see Friston et al., 1995b) where these designs can be divided into: (i) subtractive, (ii) parametric, and (iii) factorial. Parametric designs include studies where some physiological, clinical, cognitive, or sensorimotor parameter is correlated with physiology to produce an SPM of the significance of the correlation. In activation studies this may be the frequency of stimulus presentation (Price et al., 1992), time on target during a visuomotor tracking paradigm (Grafton et al., 1993), or performance on free recall (Grasby et al., 1994). Factorial designs provide the opportunity to consider an interaction between the treatments or sorts of condition (two factors interact if the level of one factor affects the effect of the other; at its simplest an interaction is a difference in a difference). In activation studies this may be the interaction between motor activation (one factor) and time (the other factor), an interaction that provides information about adaptation (Friston et al., 1992a). Another example of an interaction is between cognitive activation and the effects of a centrally acting drug (Friston et al., 1992b, Grasby et al., 1993). In this paper we have proposed that factorial designs are more powerful than subtraction designs in the analysis of cognitive processes or components. This is because the interaction between various cognitive components (factors) is explicitly included in factorial analyses and this eschews pure insertion. In particular we suggest that the effect of a cognitive component (this is independent of all other components) is best captured by the main effect of that component and that the integration of various components (i.e., the expression of one cognitive process in the context of another) is embedded in the interaction terms. Brain regions can be functionally specialized for this integration in the sense that they 
can demonstrate significant interactions in terms of their physiological responses. If we are right, then brain activations are only part of the story in mapping cognitive anatomy. Regionally specific interactions may hold the key for a more complete and richer characterization.

\section{ACKN O WLEDG MENTS}

The authors were funded by the Wellcome Trust. We thank colleagues for invaluable conversations and ideas, particularly Tim Shallice, Stephen Kosslyn, Richard Wise, Chris Frith, and Mick Rugg. We are also grateful to the two anonymous reviewers for substantial and helpful comments.

\section{REFERENCES}

Aertsen, A., and Preissl, H. 1991. Dynamics of activity and connectivity in physiological neuronal networks. In Non Linear Dynamics and Neuronal Networks (H. G. Schuster, Ed.), pp. 281-302. VCH, New York.

Corbetta, M., Miezin, F. M., Dobmeyer, S., Shulman, G. L., and Petersen, S. E. 1991. Selective and divided attention during visual discrimination of shape, color and speed: Functional anatomy by positron emission tomography. J . Neurosci. 11(8):2383-2402.

De Renzi, E., Zambolin, A., and Crisi, G. 1987. The pattern of neuropsychological impairment associated with left posterior cerebral artery territory infarcts. Brain 110:1088-1116.

Desimone, R., Albright, T. D., Gross, C. G., and Bruce, C. 1984. Stimulus selective properties of inferior temporal neurons in the macaque. J . Neurosci. 4:2051-2062.

Friston, K. J ., Frith, C., Passingham, R. E., Liddle, P., and Frackowiak, R. S. J . 1992a. Motor practice and neurophysiological adaptation in the cerebellum: A positron tomography study. Proc. R. Soc. London B 248:223-228.

Friston, K. J ., Grasby, P., Bench, C., Frith, C., Cowen, P., Little, P., Frackowiak, R. S. J ., and Dolan, R. 1992b. Measuring the neuromodulatory effects of drugs in man with positron tomography. Neurosci. Lett. 141:106-110.

Friston, K. J ., Ungerleider, L. G., J ezzard, P., and Turner R. 1995a. Characterizing modulatory interactions between areas V1 and V2 in human cortex: A new treatment of functional MRI data. Hum. Brain Map. 2:211-224.

Friston, K. J ., Holmes, A. P., Worsley, K. J ., Poline, J .-B., Frith, C. D., and Frackowiak, R. S. J. 1995b. Statistical parametric maps in functional imaging: A general linear approach. Hum. Brain Map. 2:189-210.
Friston, K. J ., Ashburner, J ., Poline, J . B., Frith, C. D., Heather, J . D., and Frackowiak, R. S. J . 1996. Spatial realignment and normalization of images. Hum. Brain Map. 3(3):165-189.

Grafton, S., Mazziotta, J ., Presty, S., Friston, K. J ., Frackowiak, R. S. J., and Phelps, M. 1992. Functional anatomy of human procedural learning determined with regional cerebral blood flow and PET. J . Neurosci. 12:2542-2548.

Grasby, P., Friston, K. J ., Bench, C., Cowen, P., Frith, C., Liddle, P., Frackowiak, R. S. J., and Dolan, R. 1992. Effect of the 5-HT1 partial agonist buspirone on regional cerebral blood flow in man. Psychopharmacology 108:380-386.

Hodges, J. R., Patterson, K., Oxbury, S., and Funnell, E. 1992. Semantic dementia: Progressive fluent aphasia with temporal lobe atrophy. Brain 115:1783-1806.

Nielsen, J. M. 1946. Agnosia, Apraxia, Aphasia: Their Value in Cerebral Localization, 2nd ed. Hoebner, New York.

Perret, D. I., Mistlin, A. J ., Potter, D. D., Smith, P. A. J ., Head, A. S., Chitty, A. J ., Broenimann, R., Milner, A. D., and J eeves, M. A. 1986. Functional organization of visual neurones processing face identity. In Aspects of Face Processing (H. Ellis, M. A. J eeves, F. Newcombe, and A. W. Young, Eds.), pp. 187-198. Nijhoff, Dordrecht.

Petersen, S. E., Fox, P. T., Posner, M. I., Mintun, M., Raichle, M. E. 1989. Positron emission tomographic studies of the processing of single words. J . Cognit. Neurosci. 1:153-170.

Price, C. J., and Humphreys, G. W. 1993. Attentional dyslexia: The effect of co-occurring deficits. Cognit. Neuropsychol. 10:569592.

Price, C. J ., Wise, R. J . S., Ramsay, S., Friston, K. J ., Howard, D., Patterson, K., and Frackowiak, R. S. J . 1992. Regional response differences within the human auditory cortex when listening to words. Neurosci. Lett. 146:179-182.

Sergent, J ., Ohta, S., and MacDonald, B. 1992. Functional anatomy of face and object processing. Brain 115:15-36.

Sternberg, S. 1969. The discovery of processing stages: Extension of Donders method. Acta Psychol . 30:276-315.

Talairach, J ., and Tournoux, P. 1988. A Co-planar Stereotaxic Atlas of a Human Brain. Thieme, Stuttgart.

Treisman, A., and Souther, J . 1985. Search asymmetry: A diagnostic for preattentive processing of separable features. J . Exp. Psychol. Gen. 114:285-310.

Ungerleider, L. G., and Mishkin, M. 1982. Two cortical visual systems. In Analysis of Visual Behaviour (D. J. Ingle, M. A. Goodale, and R. J. W. Mansfield, Eds.), pp. 549-586. MIT Press, Cambridge, MA.

Zeki, S., Watson, J . D. G., Lueck, C. J ., Friston, K. J ., Kennard, C., and Frackowiak, R. S. J . 1991. A direct demonstration of functional specialisation in human visual cortex. J . Neurosci. 11:641-649. 